\title{
Structural characterization of the PTG and PTG/PP1 complex
}

\author{
Marta Stefania Semrau ${ }^{1,2}$, Graziano Lolii ${ }^{1}$, Paola Storici' $^{2}$, Gabriele Giachin $^{3}$, Sonia Covaceuszach ${ }^{4}$ \\ ${ }^{1}$ University of Trento, Department of Cellular, Computational and Integrative Biology (CIBIO), Trento, Italy.; \\ ${ }^{2}$ Protein Facility, Elettra Sincrotrone Trieste S.C.p.A, Italy; \\ ${ }^{3}$ University of Padova, Department of Chemical Sciences (DiSC), Padova, Italy; ${ }^{4}$ CNR - Institute of Crystallography, Trieste, Italy
}

PTG (protein targeting to glycogen) is a scaffolding protein that is involved in the activation of glycogen synthesis by bringing PP1 (type 1 protein phosphatase) to its substrates. It is proposed as a target for the treatment of Lafora disease (LD), a genetic disorder manifested by catastrophic teenage onset of progressive myoclonus epilepsy. In healthy neurons, PTG is downregulated by the laforinmalin complex resulting in very low glycogen production. Mutations in malin or laforin causes accumulation of PTG, which promotes glycogen synthesis by directing PP1 to glycogen synthase and glycogen phosphorylase. This results in the appearance of neurotoxic inclusion bodies formed by insoluble polyglucosans called Lafora bodies (LB), which are ultimately responsible for Lafora disease (LD). In LD mice models knocking out PTG resulted in a nearly complete disappearance of LB and resolution of neurodegeneration and myoclonic epilepsy, indicating that small molecules interfering with the PTG/PP1 interaction emerge as an excellent therapeutic strategy for LD. Up to date, there was no structural data of PTG and PTG/PP1 complex allowing for identification of potential druggable pockets. We were able to set up expression, purification and crystallization protocols of different constructs of PTG and PTG/PP1 complex for structural studies. Here we present preliminary crystallographic structures of PTG carbohydrate binding module 21 (CBM21) and PTG/PP1 complex, complemented with SAXS analysis of the complex, that will provide a valid information for the rational design of selective compounds targeting interaction of PTG with its partners.

Keywords: lafora disease, protein targeting to glycogen (PTG), protein phosphatase 1 (PP1), The carbohydrate binding type21 (CBM21), glycogen targeting 